\title{
Corynebacterium amycolatum Causing Breast Abscess: An Infecting Diphtheroid with A Difference
}

\author{
Hena Butta ${ }^{1 *}$, Feroz Pasha ${ }^{2}$, Reetika Dawar ${ }^{1}$, Vikas Kashyap ${ }^{3}$, Leena Mendiratta ${ }^{1}$, Upasana Bora ${ }^{1}$ and Raman Sardana ${ }^{1}$ \\ Department of Microbiology, Indraprastha Apollo Hospitals, New Delhi, India \\ Department of Surgical Oncology, Indraprastha Apollo Hospitals, New Delhi, India \\ Department of Pathology, Indraprastha Apollo Hospitals, New Delhi, India
}

\begin{abstract}
Corynebacterium amycolatum has been rarely reported from cases of breast abscess/mastitis. We describe a case of Corynebacterium amycolatum causing breast abscess with fistula formation. The identification of the organism was done by MALDI- TOF (Matrix assisted Laser Desorption Ionization Time of Flight) Vitek MS (Mass spectrometry) [Biomerieux, France] and Vitek-2 (Biomerieux, France). The clinical significance of the organism was ascertained in view of presence of many polymorphonuclear cells along with Gram positive bacilli on Gram stain examination. The patient was successfully managed with surgical treatment followed by antimicrobial therapy.
\end{abstract}

\section{Keywords: Corynebacterium amycolatum, Breast Abscess, Diphtheroid}

\section{Introduction}

Non diphtheriae Corynebacterium species (Diphtheroids) constitute the normal flora of skin and upper respiratory tract and are commonly isolated as colonizer or contaminant but have also been isolated as opportunistic pathogens. The commonly isolated species in clinical microbiology laboratory are $C$. jeikeium, $C$. glucuronolyticum, C. aurimucosum, C. amycolatum, C. striatum, C. pseudodiphtheriticum, C. urealyticum, and $C$. tuberculostearicum. ${ }^{[1]}$ The identification of these organisms to species level is difficult in a routine clinical Microbiology laboratory and thus they may be regarded as contaminants even if clinically significant. Although Corynebacterium species have been found to be isolated from patients with inflammatory breast disease particularly from cases of Granulomatous lobular mastitis but the reports of Corynebacterium amycolatum causing breast infections are scarce or under-reported. ${ }^{[2,3]}$ Here, we report a case of breast abscess due to non diphtheriae Corynebacterium which was identified as $C$. amycolatum by MALDI-TOF (Matrix Assisted Laser Desorption Ionization-Time of Flight) Vitek MS (Mass Spectrometry) [Biomerieux, France] and Vitek-2 (Biomerieux, France).

\section{Case Report}

A 35 years old female, resident of Bangladesh, presented with purulent discharge from left nipple and hardness of breast. Patient had a history of lactational mastitis followed by left breast abscess for which she was operated there one year back. The patient was non-diabetic and otherwise immunocompetent with no past history of any other illness or long term medication. On local examination, there was purulent discharge from periareolar area with hard lump in central and left upper outer quadrant. The systemic examination was within normal limits. Total leucocyte count was $15,500 / \mathrm{mm}^{3}$ with $73 \%$ neutrophils and $22 \%$ lymphocytes. Excision of fistulous tract and chronic inflamed tissue was done under general anaesthesia and pus and tissue specimens were sent for microbiological and histopathological examination respectively. The histopathologic examination was suggestive of duct ectasia with dense acute on chronic mastitis, and foci of suppurative inflammation. It showed several large neutrophilic micro abscesses and collections of foamy histiocytes and foreign body type multinucleated histiocytic giant cells within the background inflammatory infiltrate. Occasional small loosely formed epithelioid cell granulomas with Langhan's type giant cells and devoid of central necrosis were also seen immediately around the ducts. Associated pathology like epithelial hyperplasia, ductal carcinoma in situ (DCIS), malignancy or tuberculosis was not seen. On Gram stain examination of pus, Gram positive bacilli and plenty of polymorphonuclear cells (PMNs) were seen (Figure-1). AFB (Acid fast bacilli) stain and fungal smear $(\mathrm{KOH})$ examination were negative. Aerobic bacterial culture showed growth of dry, white opaque colonies on Columbia sheep blood agar and these were identified as Corynebacterium amycolatum by both MALDI TOF Vitek MS and Vitek-2 ANC card (Biomerieux, France). The antimicrobialsusceptibilitytestingwasdonebydiscdiffusion 
method on $5 \%$ sheep blood agar and interpretation was done using CLSI guidelines applicable to Staphylococcus aureus ATCC25923.The organism was found to be susceptible to Amoxycillin + clavulanic acid, tetracycline, ciprofloxacin, vancomycin and chloramphenicol. Patient was initially put on oral Cefuroxime 500mg BD which was changed to Amoxycillin + clavulanic acid 1000mg (875/125mg) BD and Doxycycline $200 \mathrm{mg}$ OD for seven days following the culture report. Patient showed good response to treatment and recovered completely.

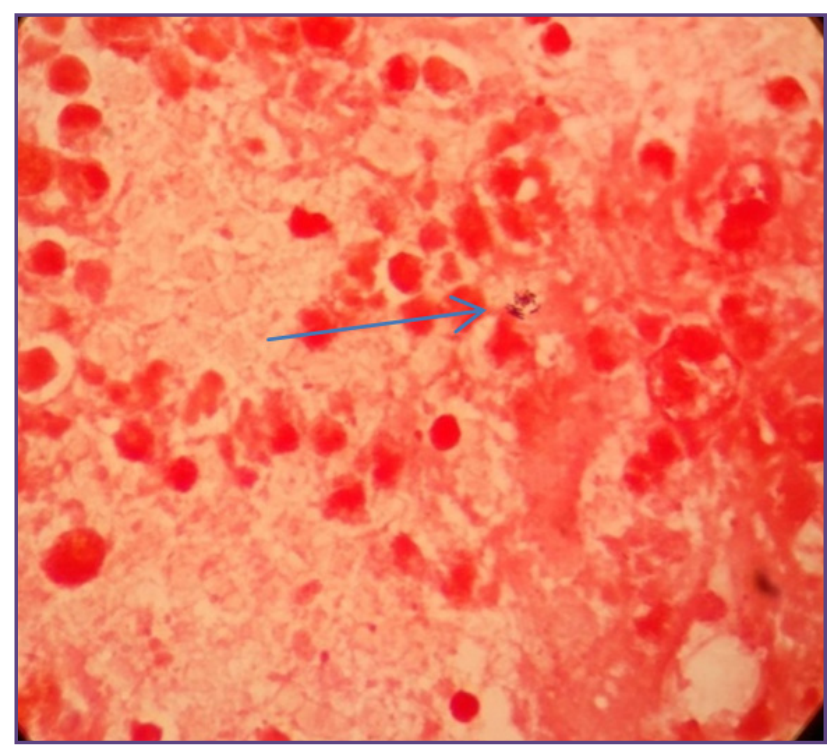

Fig. 1: Gram stain examination (1000X)- showing many pus cells and Gram positive bacilli lying in palisade.

\section{Discussion}

We have isolated clinically significant $\mathrm{C}$. amycolatum from a case of breast abscess with fistula which was successfully treated with surgical drainage and excision of fistula tract followed by antimicrobial therapy. C. amycolatum is an aerobic or facultative anaerobic non spore forming, Gram positive bacilli and is amongst the few Corynebacterium species which lack mycolic acid in their cell wall. ${ }^{[4]}$ It was first isolated from clinical specimens in 1988 and has been found to be distantly related to other Corynebacteria. ${ }^{[5]}$ C. amycolatum has been isolated from significant human infections like surgical wound infections, pilonidal sinus, mastitis, native valve and nosocomial endocarditis and septic arthritis. ${ }^{[6]}$ The case reports of breast infections due to Corynebacterium amycolatum are scarce. It may be because of the fact that Diphtheroids commonly exists as natural flora on skin and mucous membranes and their identification is difficult. So, these are not speciated further and are recognized as colonizer or contaminant. In an
Indian study by Reddy et al, C. amycolatum was found to be the most commonly occurring Corynebacterium amongst clinically significant Non diphtheria Corynebacterium. ${ }^{[4]}$ Ojaydin I et al reported a case of recurrent breast abscess by C. amycolatum. ${ }^{[7]}$ Paviour $\mathrm{S}$ et al in their retrospective study found maximum isolation of C. kroppenstedtii ${ }^{[14]}$ followed by $C$. amycolatum(Three) from breast tissue, pus or deep wound swabs of 24 women. In literature, breast tissue infections due to $C$. kroppenstedtii has been reported more frequently in comparison to $C$. amycolatum..$^{[2,8]}$ This may be because of the lipophilic nature of $C$. kroppenstedtii.C. accolens which is also lipophilic has also been reported from case of breast abscess associated with granulomatous mastitis. ${ }^{[2,9]}$ It has been stated that the significance of Corynebacteria can be determined by the presence of Gram positive bacilli along with PMNs in the clinical sample. ${ }^{[10]}$ The occurrence of Gram positive bacilli along with many PMNs and pure growth of Corynebacterium amycolatumin our case strongly signifies this organism to be the only causative pathogen for the infection. It is also interesting to note that female gender can also be a risk factor in infections due to $C$. amycolatum. Female gender has also been reported as a statistically significant species specific risk factor for C. amycolatum endocardial infections. ${ }^{[11]}$

\section{Conclusion}

We appraise Corynebacterium amycolatum as a significant cause of breast abscess even in immunocompetent patient. Direct microscopic examination of the clinical specimen and accurate identification of the isolate along with clinical details is very crucial for the correct diagnosis, appropriate antimicrobial treatment and thus optimum management of infections caused by these bacteria. MALDI TOF Vitek MS is a very useful tool for the rapid identification of these isolates.

\section{References:}

1. Williams DY, Selepak ST, Gill VJ. Identification of clinical isolates of nondiphtherial Corynebacterium species and their antibiotic susceptibility patterns. Diagn Microbiol Infect Dis $1993 ; 17: 23-8$

2. Paviour S, Musaad S, Roberts S, et al. Corynebacterium species isolated from patients with mastitis. Clinical Infectious Diseases 2002;35:1434-40.

3. Zhou F, Yu LX, Ma ZB et al. Granulomatous lobular mastitis. Chronic diseases and translational Medicine 2016;2:17-21.

4. Reddy BS, Chaudhury A, Kalawat U, Jayaprada R et al. Isolation, speciation, and antibiogram of clinically relevant 
non-diphtherial Corynebacteria (Diphtheroids). Indian $\mathrm{J}$ Med Microbiol 2012;30:52-7.

5. Mohammadi NS, Mafakheri S, Abdali N et al. Identification and characterization of the channel-forming protein in the cell wall of Corynebacterium amycolatum. Biochemica et Biophysica Acta 2013;1828:2574-82.

6. Winn WC, Allen SD, Janda WM, Koneman EW, et al. Koneman' s Color Atlas and Textbook of Diagnostic Microbiology. 6th ed. USA: Lippincott Williams and Wilkins;2006. Aerobic and facultative Gram- positive bacilli.p.765-876.

7. Ozaydin I, Yildirim M, Sahin I et al. Recurrent Breast Abscess Caused by Corynebacteriumamycolatum: A Case
Report. Turk J Med Sci 2009;39:147-9.

8. Tauch A, Fernandez-Natal I, Soriano F. A microbiological and clinical review on Corynebacterium kroppenstedtii. Int J Infect Dis 2016;48:33-9.

9. Ang LMN, Brown H. Corynebacterium accolens isolated from Breast Abscess: Possible association with Granulomatous mastitis. J Clin Microbiol 2007;45:1666-8.

10. Funke G, Von Graevenitz A, Clarridge JE 3rd, Bernard KA. Clinical Microbiology of coryneform bacteria. Clin Microbiol Rev 1997;10:125-59.

11. Belmares J, Detterline S, Pak JB and Parada JP. Corynebacterium endocarditis species specific risk factors and outcomes. BMC Infect Dis 2007;7:4.

*Corresponding author:

Dr Hena Butta, Department of Microbology, Indraprastha Apollo Hospitals, New Delhi-110076

Phone: +91 8447233605

Email: henavasdeva@yahoo.com

Date of Submission : 02.04.2017

Date of Acceptance : 17.05.2017

Financial or other Competing Interests: None.

Date of Publication : 25.08.2017 